\title{
Evaluación de la actividad de control en la contratación pública en los procesos de emergencia
}

\author{
Evaluation of the control activity in public contracting in emergency processes
}

Avaliação da atividade de controle em compras públicas em processos de emergência

César Augusto Bedón Salzar
cebedon.sa@gmail.com
https://orcid.org/0000-0003-3751-2504

Guido Escobar Pérez

gigescobar@hotmail.com

https://orcid.org/0000-0002-1654-5203

\section{Pontifica Universidad Católica del Ecuador Sede Ambato, Latacunga-Ecuador}

Artículo recibido en julio 2021 | Arbitraje en agosto 2021 | Aceptación en septiembre 2021 | Publicación en octubre 2021

RESUMEN

ABSTRACT

RESUMO
El Órgano técnico de control durante emergencia evidencia riesgos de corrupción, sin embargo, las contradicciones, arbitrariedades del procedimiento generan hallazgos viciados, e ilegales; que no mejoran los controles internos, ni reducen el riesgo, y vician los derechos y garantías del debido proceso de los auditados. Dado lo expuesto, la investigación tiene como objetivo evaluar el procedimiento y la actividad de control en la contratación pública de emergencia, para la disminución de las arbitrariedades que afectan la gestión. La metodología que se emplea es teórica-práctica, dado que, permite confirmar los errores del control en el campo de la legalidad, a partir del enfoque cualitativo, que se aplica para identificar las causas de la arbitrariedad en la acción de control. Los resultados, se enmarcan en el control de los procedimientos de contratación, cuya aplicación facilita la reducción de la irracionabilidad y arbitrariedad en los informes aprobados por Contraloría General del Estado.

Palabras clave: Arbitrariedad; Control; Contratación por emergencia; Debido proceso; Eficiencia

The technical control body during emergency evidences corruption risks, however, the contradictions and arbitrariness of the procedure generate flawed and illegal findings, which do not improve internal controls, nor reduce the risk, and vitiate the rights and guarantees of due process of the auditees. Given the above, the purpose of this research is to evaluate the procedure and the control activity in emergency public contracting, in order to reduce the arbitrariness that affects management. The methodology used is theoretical-practical, since it allows confirming the control errors in the field of legality, from the qualitative approach, which is applied to identify the causes of arbitrariness in the control action. The results are framed in the control of contracting procedures, the application of which facilitates the reduction of irrationality and arbitrariness in the reports approved by the State Comptroller General's Office.

Key words: Arbitrariness; Control; Emergency Contracting; Due Process; Efficiency

O órgão de controle técnico durante emergências evidencia riscos de corrupção, entretanto, as contradições e arbitrariedades do procedimento geram conclusões errôneas e ilegais, que não melhoram os controles internos, nem reduzem os riscos, e viciam os direitos e garantias do devido processo dos auditados. Dado o acima exposto, o objetivo da pesquisa é avaliar o procedimento e a atividade de controle nas compras públicas emergenciais, a fim de reduzir a arbitrariedade que afeta a gestão. A metodologia utilizada é teórico-prática, pois permite a confirmação de erros de controle no campo da legalidade, com base na abordagem qualitativa, que é aplicada para identificar as causas de arbitrariedade na ação de controle. Os resultados são enquadrados no controle dos procedimentos de contratação, cuja aplicação facilita a redução da irracionalidade e da arbitrariedade nos relatórios aprovados pela Controladoria Geral de Contas do Estado.

Palabras clave: Arbitrariedade; Controle; Contratação de emergência; Processo justo; Eficiência 
INTRODUCCIÓN

El control gubernamental que aplica Contraloría General del Estado en la emergencia sanitaria COVID-19, evidencia riesgos de fraude, que, se traducen en actos de corrupción, sin embargo, las contradicciones, arbitrariedades y la inobservancia del elemento de procedibilidad para el control posterior de los procesos de compras públicas, especialmente, en los contratos de emergencia, generan recomendaciones en los informes aprobados por el Órgano de Control, que son calificadas, como: ilegales, extemporáneas e imposibles de aplicar, con lo cual, no se mejoran los controles internos, ni se alcanza el objetivo de reducción del riego de fraude. Así mismo, dadas las arbitrariedades del procedimiento, se vician los derechos y garantías del debido proceso de los sujetos activos de la acción de control, es decir, los servidores públicos auditados.

La investigación tiene como objetivo evaluar el procedimiento y la actividad de control en la contratación pública en los procesos de emergencia, para la disminución de las arbitrariedades que afectan la gestión.

MÉTODO

La metodología que se emplea es teórica-práctica, dado que, permite confirmar los errores del control en el campo de la legalidad, a partir del enfoque cualitativo, que se aplica para identificar las causas de la arbitrariedad e improcedibilidad de la acción de control. Los resultados, se enmarcan en el control de los procedimientos de contratación por emergencia, cuya aplicación facilita la reducción de la irracionabilidad y arbitrariedad en los informes aprobados por Contraloría General del Estado Ecuatoriano.

RESULTADOS

\section{Elementos de referencia procesal para el control de la contratación situación de emergencia}

Con estos elementos, a fin de que el proceso de control gubernamental aplicado a los procesos de contratación bajo situación de emergencia ejecutados por los equipos de control de la Contraloría General del Estado no sufra de falencias del debido proceso que atenten con las garantías y derechos de los auditados, los equipos de control advertirán los siguientes elementos procedimentales que se aprecian en la Tabla 1. 
Tabla 1. Debido proceso control gubernamental procesos contratación situación de emergencia.

\begin{tabular}{|c|c|c|c|}
\hline ETAPA & PROCEDIMIENTO & RESPONSABLE & ACCIÓN \\
\hline 1 & $\begin{array}{l}\text { NOTIFICACIÓN DE } \\
\text { INFRACCIÓN PROCESO } \\
\text { CONTRTACIÓN } \\
\text { PÚBLICA }\end{array}$ & $\begin{array}{l}\text { SERVICIO NACIONAL DE } \\
\text { CONTRATACIÓN PÚBLICA }\end{array}$ & $\begin{array}{l}\text { EVALUACIÓN DEL PROCESO } \\
\text { PRECONTRACTUAL Y CONTRACTUAL, } \\
\text { REVISIÓN CUMPLIMIENTO NORMATIVO Y } \\
\text { PROCEDIMIENTO }\end{array}$ \\
\hline 2 & $\begin{array}{l}\text { MODIFICACIÓN PLAN } \\
\text { ANUAL DE CONTROL }\end{array}$ & $\begin{array}{l}\text { CONTRALORÍA GENERAL } \\
\text { DEL ESTADO }\end{array}$ & $\begin{array}{l}\text { RESOLUCIÓN SUBCONTRALOR GENERAL } \\
\text { DEL ESTADO REFORMA PLAN ANUAL DE } \\
\text { CONTROL }\end{array}$ \\
\hline 3 & $\begin{array}{l}\text { EMISIÓN DE ORDEN DE } \\
\text { TRABAJO }\end{array}$ & $\begin{array}{l}\text { CONTRALORÍA GENERAL } \\
\text { DEL ESTADO }\end{array}$ & \\
\hline 4 & $\begin{array}{l}\text { NOTIFICACIÓN DE } \\
\text { INICIO }\end{array}$ & $\begin{array}{l}\text { JEFE DE EQUIPO DE } \\
\text { AUDITORIA }\end{array}$ & $\begin{array}{l}\text { NOTIFICACIÓN EN PERSONA A TODOS } \\
\text { LOS SERVIDORES REACIONADOS, ASÍ } \\
\text { COMO EX SERVIDORES. }\end{array}$ \\
\hline 5 & $\begin{array}{l}\text { REQUERIMIENTO DE } \\
\text { INFORMACIÓN }\end{array}$ & $\begin{array}{l}\text { JEFE DE EQUIPO DE } \\
\text { AUDITORIA }\end{array}$ & $\begin{array}{l}\text { SOLICITAR POR ESCRITO, EXPEDIENTES } \\
\text { ADMINISTRATIVOS Y CONTRACTUALES } \\
\text { DE SITUACIÓN DE EMERGENCIA }\end{array}$ \\
\hline 6 & $\begin{array}{l}\text { VERIFICACIÓN } \\
\text { RESOLUCIÓN DE } \\
\text { EMERGENCIA }\end{array}$ & $\begin{array}{l}\text { FIRMADO MÁXIMA } \\
\text { AUTORIDAD O DELEGADA/O }\end{array}$ & $\begin{array}{l}\text { REVISAR SU PUBLICACIÓN, ALCANCE } \\
\text { (ACONTECIMIENTO GRAVE) FUERZA } \\
\text { MAYOR O CASO FORTUITO, A NIVEL } \\
\text { NACIONAL, SECTORIAL O INSTITUCIONAL }\end{array}$ \\
\hline \multirow[t]{8}{*}{7} & $\begin{array}{l}\text { ANÁLISIS EXPEDIENTE } \\
\text { CONTRACTUAL }\end{array}$ & EQUIPO DE CONTROL & $\begin{array}{l}\text { VERIFICAR EXISTENCIA PREVIA } \\
\text { CERTIFICACIÓN PRESUPUESTARIA }\end{array}$ \\
\hline & & & $\begin{array}{l}\text { ESTUDIO DE MERCADO /DETERMINAR EL } \\
\text { VALOR ACTUALIZADO BIEN, SERVICIO, } \\
\text { OBRA - ESTUDIO PREVIO (RELACIONADA } \\
\text { CON LA SITUACIÓN DEMEMERGENCIAY } \\
\text { QUE PERMITAN SUPERARLA) }\end{array}$ \\
\hline & & & $\begin{array}{l}\text { RESOLUCIÓN DE INICIO PROCESO } \\
\text { PRECONTRACTUAL (NECESIDAD ACTUAL } \\
\text { Y EMERGENTE) }\end{array}$ \\
\hline & & & $\begin{array}{l}\text { NO REQUIERE PLIEGOS - DOCUMENTOS } \\
\text { PRECONTRACTUALES }\end{array}$ \\
\hline & & & $\begin{array}{l}\text { INVITACIÓN A PROVEEDOR/ES (NO RUP) } \\
\text { SI RUC }\end{array}$ \\
\hline & & & $\begin{array}{l}\text { OFEENTE NO ESTAR INCUROS } \\
\text { INHABILIDADES GENERAL Y/O ESPECIAL }\end{array}$ \\
\hline & & & $\begin{array}{l}\text { ACTA DE ANÁLISIS OFERTA (MEJOR } \\
\text { PRECIO - NO NECESARIAMENTE COSTO) }\end{array}$ \\
\hline & & & RESOLUCIÓN DE ADJUDICACIÓN \\
\hline
\end{tabular}




\begin{tabular}{|c|c|c|c|}
\hline ETAPA & PROCEDIMIENTO & RESPONSABLE & ACCIÓN \\
\hline 8 & $\begin{array}{l}\text { ANALSIS RESOLUCIÓN } \\
\text { CIERRE Y/O } \\
\text { SUPERACIÓN DE } \\
\text { EMERGENCIA }\end{array}$ & EQUIPO DE CONTROL & $\begin{array}{l}\text { LAS ADQUISICIONES Y CONTRATACIONES } \\
\text { REALIZADAS PERMITAN SUPERAR LA } \\
\text { SITUACIÓNDE EMERGENCIA, ASII COMO } \\
\text { MITIGAR LOS EFECTOS PRODUCIDOS POR } \\
\text { EL EVENTO O ACONOTECIMIENTO GRAVE }\end{array}$ \\
\hline 9 & $\begin{array}{l}\text { NOTIFICACIÓN } \\
\text { RESULTADOS } \\
\text { PROVISIONALES }\end{array}$ & $\begin{array}{l}\text { JEFE DE EQUIPO DE } \\
\text { AUDITORIA }\end{array}$ & $\begin{array}{l}\text { DE MANERA FÍSICA, Y/O CORREO } \\
\text { ELECTRÓNICO SEÑALADO EN LA } \\
\text { RESPUESTA DE NOTIFICACIÓN DE INICIO. } \\
\text { SE DEBE PONER EN CONOCIMIENTO } \\
\text { LOS HALLAZGOS OBTENIDOS LUEGO DE } \\
\text { APICAR LAS PRUEBAS DE CAMPO DE LA } \\
\text { ACCIÓN DE CONTROL }\end{array}$ \\
\hline 10 & $\begin{array}{l}\text { NOTIFICACIÓN } \\
\text { CONVOCATORIA } \\
\text { AUDIENCIA DE } \\
\text { LECTURA DE } \\
\text { BORRADOR }\end{array}$ & $\begin{array}{l}\text { JEFE DE EQUIPO DE } \\
\text { AUDITORIA }\end{array}$ & $\begin{array}{l}\text { DE MANERA FÍSICA, Y/O CORREO } \\
\text { ELECTRÓNICO SEÑALADO EN LA } \\
\text { RESPUESTA DE NOTIFICACIÓN DE INICIO. }\end{array}$ \\
\hline 11 & $\begin{array}{l}\text { ENTREGA DE } \\
\text { BORRADORES DE } \\
\text { INFORME }\end{array}$ & $\begin{array}{l}\text { JEFE DE EQUIPO DE } \\
\text { AUDITORIA }\end{array}$ & POSTERIOR A LA LECTURA HASTA 5 DÍAS \\
\hline 12 & $\begin{array}{l}\text { ANÁLISIS DE } \\
\text { RESPUESTAS } \\
\text { PERSONAS } \\
\text { RELACIONADAS } \\
\text { BORRADOR DE } \\
\text { INFORME }\end{array}$ & EQUIPO DE CONTROL & $\begin{array}{l}\text { PREVIO A LA EMISIÓN DEL INFORME } \\
\text { FINAL Y TRAMITE DE APROBACIÓN }\end{array}$ \\
\hline 13 & $\begin{array}{l}\text { APROBACIÓN INFORME } \\
\text { FINAL }\end{array}$ & $\begin{array}{l}\text { MAXIMA AUTORIDAD } \\
\text { CONTROL }\end{array}$ & $\begin{array}{l}180 \text { DÍAS POSTERIORES A LA EMISIÓN A } \\
\text { LA ORDEN DE TRABAJO. }\end{array}$ \\
\hline
\end{tabular}

Fuente: Modificado el 3 de septiembre de 2021.

\section{Reforma del Subsistema Nacional de Control}

Conforme a las observaciones y falencias procedimentales advertidas, las cuales se ubican entre los actores activos y pasivos del Sistema Nacional de Contratación Pública y del Sistema Gubernamental de Control, es necesario que estos se fusionen en el marco de los principios de coordinación $n^{1}$ y colaboración ${ }^{2}$, por ello es imperativo que se redefina el objeto del Subsistema 1 Principio de coordinación. Las administraciones públicas desarrollan sus competencias de forma racional y ordenada, evitan las duplicidades y las omisiones.

2 Principio de colaboración. Las administraciones trabajarán de manera coordinada, complementaria y prestándose auxilio mutuo. Acordarán mecanismos de coordinación para la gestión de sus competencias y el uso eficiente de los recursos. 
Nacional de Control, pues se advierte, que su concepción no está determinada a cumplir con la buena administración pública, en virtud que se opone fundamentalmente a los principios de legalidad, eficiencia, eficacia, calidad, transparencia, ya que, éste se basa en la irregularidad ${ }^{3}$ dentro del proceso de contratación pública.

En consecuencia, la actividad y competencias de este Subsistema debe establecer una estructura mixta de control, que identifique el control concurrente y defina competencias, basada en la lealtad institucional entre el ente rector de la contratación y el órgano técnico de control, evitando solo caer en la sanción, dejando de lado el mejoramiento continuo del proceso, esto por cuanto el legislador aprobó la creación del Subsistema con la premisa de que todo los actores y actos de la contratación pública se caracterizan por la corrupción. Por lo tanto, es pertinente que esta fusión de control permita no solo proteger el recurso público y el interés general, sino también el derecho de los ciudadanos a una buena administración pública por medio de la satisfacción de sus necesidades.

En ese contexto, se debe reformar la composición del Subsistema en sus integrantes, estableciéndose que los únicos miembros del Subsistema Gubernamental de Control, sean (SERCOP) y (CGE) para que ejercerán su competencia de asesoría conjunta, y control, tutelados por un Sistema Jurídico exclusivo para control y no de determinación de infracción como se desarrolla actualmente después de la reforma (LOSNCP), de esta manera, el Subsistema se constituirá en guía de los procedimientos y actos administrativos que tienen como objetivo el cumplimiento de los fines institucionales y la satisfacción de las necesidades de los ciudadanos, reduciendo el riegos de fraude y/o corrupción, y no convirtiéndose en un obstáculo como son también ahora los dictámenes previos de contratación de la Contraloría General del Estado.

\section{DISCUSIÓN}

\section{Elementos normativos atenta el control de la contratación en emergencia sanitaria}

Con la declaración de emergencia sanitaria, provocada por la expansión del virus SARS-CoV-2 que produce COVID-194, la Comisión Interamericana de Derechos Humanos (CIDH), mediante Resolución No. 1/20205 , fijó recomendaciones a los Estados orientadas a la atención y contención de la pandemia, además, advertido que los Gobiernos deben redefinir el cumplimiento de sus

3 Ley Orgánica del Sistema Nacional de Contratación Pública, Art. 15. - “(..) El Subsistema Nacional de Control tendrá como deber efectuar un control coordinado, articulado, interconectado, permanente y eficaz de cualquier situación o irregularidad que se presentare en la contratación pública, conforme el ámbito de competencias de cada entidad."

4 Enfermedad causada por el nuevo coronavirus conocido como SARS-CoV-2. La OMS tuvo noticia por primera vez de la existencia de este nuevo virus el 31 de diciembre de 2019, al ser informada de un grupo de casos de «neumonía vírica» que se habían declarado en Wuhan (República Popular China) https://www.who.int/es/news-room/q-a-detail/coronavirus-disease-covid-19.

5 Recuperado de: http://oas.org/es/cidh/decisiones/pdf/Resolucion-1-20-es.pdf, 12 de junio 2021. 
fines y objetivos, así como, la aplicación de políticas públicas con el propósito de priorizar el fortalecimiento estructural de la asistencia estatal en salud, esto conforme la Resolución 4/2020 $(\mathrm{CIDH})^{6}$, pedido que se hace más obligatorio en razón de la desigualdad de la concentración de la riqueza en nuestros países, así, " $1 \%$ de los ciudadanos tiene el $82 \%$ de la riqueza, por otra parte, más de 10 millones de niños menores de 5 años mueren por año por enfermedades que se pudieron prevenir, 1000 millones de personas viven con menos de 1 dólar diario" (Ávila, 2020).

En consecuencia, los Estados para cumplir estas obligaciones, deben instrumentar medidas legislativas, que permitan efectivizar y garantizar el acceso sin discriminación del derecho humano a la salud, incluido el goce a bienes y servicios eficientes de salud, para lo cual, es necesario que todos los recursos económicos sean puestos a su disposición. El objetivo debía ser prevenir serios deterioros en salubridad o afectación de las vidas de los habitantes de un país, y de esta manera evitar nuevos y mayores riesgos en los deficientes sistemas de salud pública, que se vieron colapsados?.

En ese marco, a fin de cumplir con las recomendaciones de la $\mathrm{CIDH}$, en nuestro país, durante este tiempo de emergencia sanitaria, se emitió diferentes disposiciones, así el Ejecutivo por medio de Decretos, Acuerdos y Resoluciones ministeriales; y, otras; desde la Asamblea Nacional con la denominada Ley Orgánica de Apoyo Humanitaria ${ }^{8}$ (LOAH), reformas al Código Integral Penal (COIP) y a la Ley Orgánica del Sistema Nacional de Contratación Pública9 (LOSNCP), trataron de priorizar el derecho a la salud, así como la atención para superar la situación de emergencia sanitaria. Las cuales llegaron de manera extemporánea, con relación a lo realizado por el Servicio Nacional de Contratación Pública (SERCOP), que emitió directrices y reformas a la regulación de los procesos de contratación pública por situación de emergencia, así como para el manejo administrativo de la misma, entre los cambios se advierten, las Resoluciones No. RE-SERCOP-2020-0104 de 19 de marzo de 2020, y la No. RE-SERCOP-2020-0104 de 6 de abril de 2020, así como los oficios circulares SERCOP-SERCOP-2021-0004-C; y, SERCOP-SERCOP2020-0017-C, cuyo objetivo era viabilizar la superación de la emergencia y la superación de sus efectos, es decir, constituirse en herramientas facilitadoras en los diferentes niveles de gobiernos, y funciones del sector público para atender las emergencias en sus territorios y en el marco de sus competencias, pero no consideró la disposición del artículo 57 de LOSNCP, que fueron reformadas recién en enero 2021, lo cual generó discrepancias procedimentales, que atentan a los principios generales del derecho administrativo como el c, que se oponen a los elementos de la balanza de equilibrios desarrollado por Monstesquieu (Dromi, 1979), pues las reformas constituyen más poder al Estado, en reducción de las garantías de los administrados.

6 Recuperado de: https://www.oas.org/es/cidh/decisiones/pdf/Resolucion-4-20-es.pdf, 12 de junio de 2021.

7 La evolución de la pandemia en Ecuador ya satura los hospitales en Quito, Recuperado de: https://www.opendemocracy.net/es/pandemiaecuador-satura-hospitales-quito/

$8 \quad$ Publicado en el Registro Oficial Suplemento No. 229 del 22 de junio de 2020.

9 Publicado en el Registro Oficial, Segundo Suplemento No. 392, de fecha 17 de febrero de 2021. 
Así entre las reformas contradictorias al procedimiento de emergencia, se evidencian las modificaciones a las Resoluciones No. RE-SERCOP-2016-0000072, No. RE-SERCOP-2020-0104 de 6 de abril de 2020, que se detallan en la Tabla 2.

Tabla 2. Reformas resolutivas SERCOP.

\section{RE-SERCOP-2016-0000072}

\section{RE-SERCOP-2020-0104}

Las entidades contratantes, están en la obligación de declarar de forma expresa la imposibilidad de ejecutar procedimientos de contratación comunes para adquirir B/S incluidos consultoría
Determinan plazo de la resolución de emergencia no debe ser mayor a 60 días. La excepción, es la ampliación del estado de excepción.

Entidades para a ejecutar procesos de contratación, determinaran relación directa y objetiva entre la situación de emergencia y la urgencia de la adquisición $\mathrm{B} / \mathrm{S}$ incluidos consultoría.

Entidades obligatoriamente deben contar con certificación de disponibilidad presupuestaria.

Prohibición contratación, en el caso de que el plazo de ejecución se extiende más del tiempo establecido en la declaratoria de emergencia.
Las necesidades se pueden consolidar con otros requirentes, a fin de que se realice de forma unificada un procedimiento de selección único.

Fuente: Modificado a partir de Resolución No. RE-SERCOP-2020-0104 de 6 de abril de 2020.

Otra introducción normativa fue la del 9 de abril de 2020, cuando el SERCOP, expidió la Resolución No. RE-SERCOP-2020-0105, que reformó el procedimiento de emergencia sanitaria contenida en la Resolución Externa No. RE-SERCOP-2016-0000072 (Figura 1).

En las contrataciones de emergencia cuyo objeto sea la adquisición de bienes en el extranjero, y cuya importación la realice directamente la entidad contratante, se exime de realizar la verificación de no existencia de producción u oferta nacional, así como tampoco de la autorización de importación por parte del SERCOP.

En las contrataciones necesarias para atender y superar una situación de emergencia cuyo objeto tenga relación a servicios de salud o exequiales; se dará por cumplido el análisis de la oferta existente en el mercado, cuando las entidades publiquen sus necesidades de contratación en su página web institucional.

Los contratos, órdenes de compra de emergencia o facturas generados en el marco de la declaratoria de emergencia, deberán instrumentarse por escrito, es decir, deberán estar elaborados y perfeccionados por medios físicos o electrónicos.

Figura 1. Resolución Externa No. RE-SERCOP-2016-0000072. Fuente: Modificado a partir de Resolución No. RE-SERCOP-2020-0105 de 9 de abril de 2020. 
A partir de estas reformas tanto legales como reglamentarias, el órgano rector del Sistema Nacional de Contratación Pública puso a consideración de las entidades del Estado y usuarios en general en su página www.secop.gob.ec/sercop/, como se destaca en la Figura 2.

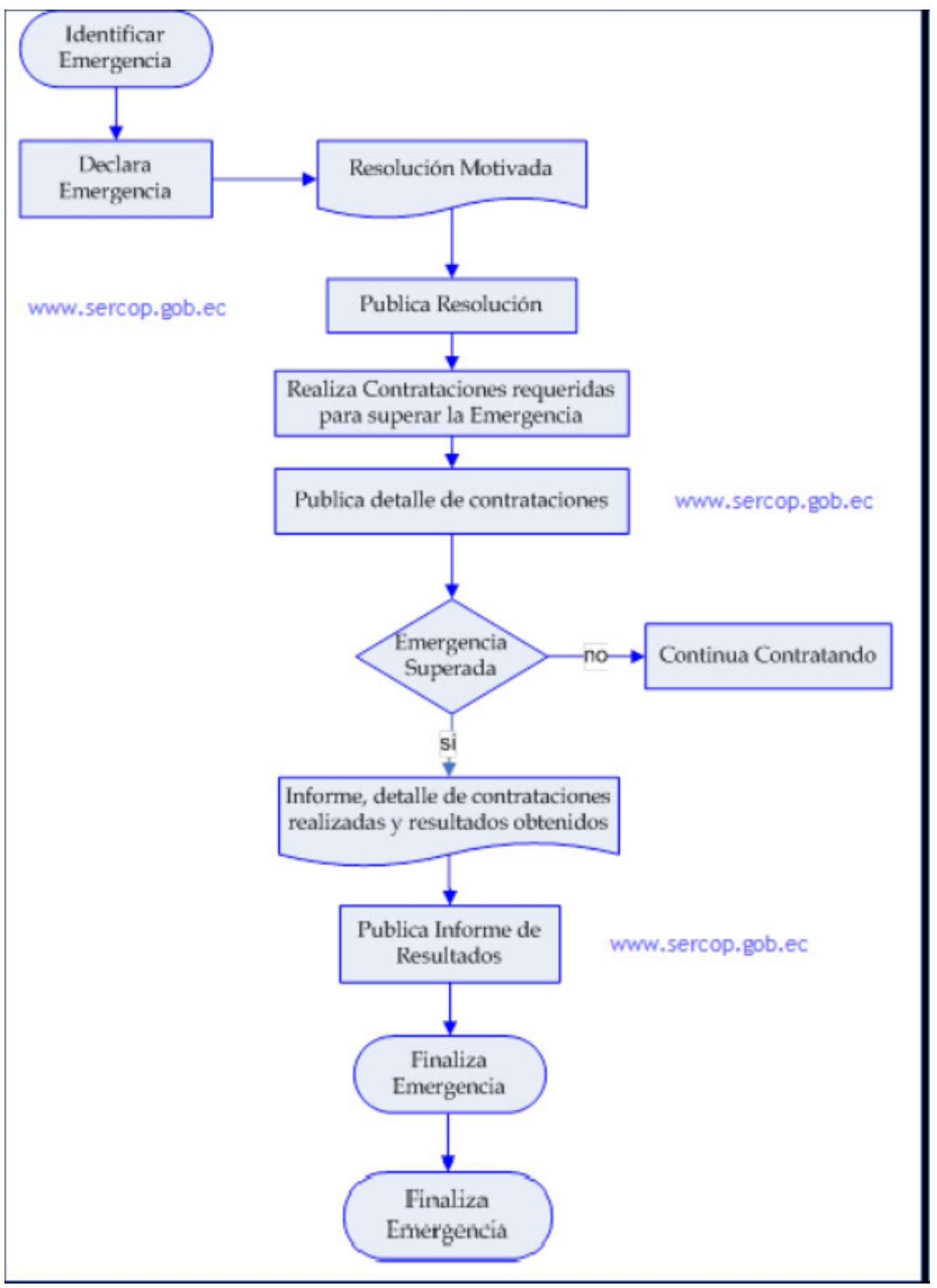

Figura 2. Diagrama de los procesos de las entidades del Estado y usuarios. Fuente: www.sercop.gob.ec 
Con estos elementos queda definido el procedimiento reglamentario, es decir, el referente procedimental, que debe ser aplicado por las entidades públicas en los procesos de contratación pública en situación de emergencia, pero, que por otra parte, debería servir como elemento de seguridad jurídica, y elemento de tipicidad; sin embargo, esta dispersión jurídica contradictoria con (LOSNCP) no cumple con la finalidad de dotar al individuo de los mecanismos de protección frente a la arbitrariedad del poder estatal (Del Pozo, 2005), es decir, que estos cambios imprevistos afectan la actuación administrativa y confrontan disposiciones normativas, constitucionales y el principio de legalidad, lo cual afecta también el control posterior.

Las reformas generan una contradicción en la actuación administrativa de los órganos estatales, pues las leyes, decretos y normas secundarias son emitidos sin concordancia, (Robanlino, 2008), por lo que se debe siempre considerar los principios generales del derecho administrativo, pues, su aplicación es la base de la potestad administrativa y por ello no puede ser utilizada de manera arbitraria por las autoridades estatales, sino debe ser el medio para el cumplimiento de los fines de la administración y garantías (Marienhoff, 1998), que se reflejan a favor del ciudadano en los procesos de control posterior que se ejecutan por la Contraloría General del Estado (Escobar, 2016)1.

Entonces, se colige que ese órgano ejerce sus competencias después de ejecutados los hechos, actos administrativos y resolutivos de las decisiones dentro de la potestad pública, lo cual es también desarrollado por la LOSNCP. En ese contexto para el ejercicio de la potestad pública de control posterior, Contraloría General del Estado, lo ejecutará cumpliendo lo dispuesto en el 8 y 15 LOSNCP2$^{2}$. En consecuencia, existe un control interno previo y/o paralelo realizado por el órgano rector del Sistema y las propias entidades públicas; y otro el control externo aplicado por órganos definidos en la norma, pero, como lo dice (Granda, 2017) ambos tipos de control deben estar coordinados para garantizar su autonomía y eficiencia de su gestión.

En ese contexto, la Contraloría General del Estado, previo a la emisión de la orden de trabajo, requiere que el (SERCOP) remita informe de infracción de la norma del Sistema Nacional de Contratación Pública artículos 15 de (LOSNCP) “(...) El ente rector del Sistema Nacional de Contratación Pública informará a la Contraloría General del Estado, (...) cada vez que conozca el cometimiento de infracciones a lo dispuesto en esta Ley. Esta información será pública y se reportará en el Portal de Compras Públicas..."; y 6 numeral 4 del Reglamento General a la Ley

1 Escobar, 2016, pág. 55 "se constituyó en un organismo superior de control, que tiene por finalidad el control externo de los recursos de las entidades del sector público; que lo efectúa mediante el examen posterior de las operaciones financieras y administrativas de cada entidad, por medio de exámenes especiales y auditoria gubernamental".

2 Ley Orgánica del Sistema Nacional de Contratación Pública, Art. 8. - Órganos competentes. -El Servicio Nacional de Contratación Pública junto con las demás instituciones y organismos públicos que ejerzan funciones en materia de presupuestos, planificación, control y contratación pública, forman parte del Sistema Nacional de Contratación Pública, en el ámbito de sus competencias.

Art. 15. -Subsistema Nacional de Control. - “(...) El ente rector del Sistema Nacional de Contratación Pública informará a la Contraloría General del Estado, (...) cada vez que conozca el cometimiento de infracciones a lo dispuesto en esta Ley. Esta información será pública y se reportará en el Portal de Compras Públicas..." 
Orgánica del Sistema Nacional de Contratación Pública, “...generar alertas o recomendaciones de cumplimiento obligatorio, sin perjuicio de que sean puestas en conocimiento de los organismos de control pertinentes".

Por lo tanto, este requisito previo se constituye no solo en elemento de procedibilidad, sino de legalidad, ya que el posible vicio o ilegalidad en el proceso de contratación puede ser subsanado dependiendo de la etapa del procedimiento y de la eventual afectación que se haya generado, ya que, estos pueden ser eliminados por medios de impugnación) (Vásquez, 2021), (reclamo administrativo, recurso de apelación; y denuncia) ${ }^{3}$; los cuales permitan el restablecimiento de la legalidad, y tomando siempre como base la pretensión del impugnante, no obstante, estos solo favorecen al oferentes; mientras que el Estado no puede ejercer el derecho de impugnación; por ende es el (SERCOP) como ente rector del sistema, y que regula la normativa del procedimiento, el único competente para emitir el instrumento vinculante para verificar, la legalidad, la gestión y los resultados de la contratación (Lara, 2012) lo cual conlleva al proceso de control, pues finalmente, está claro que por las impugnaciones, no todas las observaciones y/o infracciones en el proceso precontractual y contractual deben terminar en una acción de control.

Finalmente, se debe tener en cuenta que las adquisiciones del Estado no se las hace como agente de derecho privado, sino por la supremacía del interés general, que no solo debe buscar el mejor precio, sino la satisfacción de las necesidades de la población. Por lo tanto, compete al órgano técnico de control respetar los principios de legalidad, seguridad jurídica, interdicción de la arbitrariedad, y otros relacionados con el debido proceso y buena administración pública que rodean al proceso de auditoría.

\section{El debido proceso en el Sistema de Control Administrativo}

Por la preponderancia que se merece el control en nuestra legislación constitucional se la ubica como una de las funciones del Estado, en la de Transparencia y Control Social4; cuya conformación se estructura en diferentes órganos, entre los cuales se advierte la Contraloría General del Estado (CGE), cuya composición y funciones están desarrolladas en los artículos 211 y 212 de la Constitución de la República (CRE), y tiene concordancia con el artículo 31 de la Ley Orgánica de la Contraloría General del Estado (LOCGE), coligiendo que una función primordial es la de examinar, verificar y evaluar el cumplimiento de la visión, misión y objetivos de las instituciones del Estado en conformidad con la utilización de recursos y bienes públicos, es decir, el control y evaluación de la actividad administrativa (Marquez, 2005) citado por el

3 Vásquez, pág 7“En la contratación pública ecuatoriana tenemos expresamente reconocidos como medios de impugnación a la reclamación administrativa, al recurso de apelación y a la denuncia"

4 Art. 225 (...) 1. . Los organismos y dependencias de las funciones Ejecutiva, Legislativa, Judicial, Electoral y de Transparencia y Control Socia 
doctor Escobar5; lo cual debe cumplir con la dirección del Sistema de Control, Fiscalización y Auditoría del Estado.

Se debe entender, que el Control de manera general, es un proceso dinámico, que se basa en una relación definida entre dos sujetos, el auditado y el órgano de control. relación que se caracteriza, por una mutua desconfianza conforme lo indica (Ballén, 1998), y por una condición de desventaja del auditado frente a la administración, que se refleja en la auto-tutela ejecutiva de la administración ${ }^{6}$, como lo sustentan (Guanchez, 2012) y (Bonnemaison, 2000); y por esa condición de vulnerabilidad el sujeto auditado debe estar asistido de derechos y garantías (Dromi, 2015), que en palabras de (Escobar, 2018) jurídicamente es una herramienta que permite sujetar todo el aparato de la administración al principio de legalidad; y en virtud de este elemento, la ejecución de los actos de la administración de control deben tener límites (Marquez, 2005), a fin de evitar como lo sostiene (Valadés, 2006) la arbitrariedad, excesos de poder, contradicciones y restricción de libertades; y así, obtener una buena administración pública, evitando la violación del debido proceso bajo la perspectiva de la legitimidad (Valadés, 2006).

Por disposición constitucional y legal, Contraloría General del Estado tiene la facultad de realizar un control posterior al desarrollo de las operaciones y actividades institucionales según (Coopers y Lybrand, 1997), y por la aplicación de la Norma Técnica de Control Interno, se constituye en una responsabilidad compartida entre los servidores públicos y la auditoría. En esa línea, el control expresado en exámenes especiales ${ }^{7}$ se constituyen en actos coordinados entre la instancia de control superior y el cumpliendo de la normativa vigente (Pickett, 2007), es decir, el control se realiza en correspondencia con las leyes, regulaciones y la normativa aplicable en el tiempo, en consecuencia, en razón al menos como lo dicta (Gordillo, 2017) la aplicación del sistema de control se lo ejecutará bajo principios de legalidad, objetividad, oficialidad, informalismo a favor del administrado, debido proceso, contradicción.

Por otra parte, la identificación de los sujetos pasivos nos permite determinar el ámbito de aplicación de los procesos de control de la administración, que se encuentran definidos en el artículo 2 (LOCGE) ${ }^{8}$, revisiones que se ejecutan en razón del - Sistema de Control, Fiscalización y Auditoría del Estado - para lo cual se requieren revisiones y controles planificados, pues sin los mismos estos son imposibles (Harold Koontz y Heinz Weihrich, 2007), además, que se deben ejecutar de forma periódica, en razón de las actividades que permanente se desarrollan

5 El control Gubernamental DETERMINACIÓN DE RESPONSABILIDADES Administrativa, Civiles e Indicios Penales, pag. 26

6 Facultad de la Administración para el uso de su propia coacción sin necesidad de recabar el apoyo de los tribunales, pudiendo proceder previo apercibimiento, a la ejecución forzosa de loas actos administrativos.

7 Art. 19.- Examen Especial como parte de la auditoría gubernamental el examen especial verificará, estudiará y evaluará aspectos limitados o de una parte de las actividades relativas a la gestiónfinanciera, administrativa, operativa y medio ambiental, con posterioridad a su ejecución, aplicará las técnicas y procedimientos de auditoría, de la ingeniería o afines, o de las disciplinas específicas, de acuerdo con la materia de examen y formulará el correspondiente informe que deberá contener comentarios, conclusiones y recomendaciones

$8 \quad$ Art. 2. - Ámbito de aplicación de la Ley.- Las disposiciones de esta Ley rigen para las instituciones del sector público determinadas en los artículos 225, 315 y a las personas jurídicas de derecho privado previstas en el artículo 211 de la Constitución 
por las entidades públicas, para lo cual, el Órgano de Control lo aplica mediante auditorías gubernamentales y exámenes especiales ${ }^{9}$ que se efectúan a las entidades que integran el sector público, y personas jurídicas de derecho privado que manejan fondos públicos.

Con el nuevo pacto social, se declara que Ecuador es un Estado Constitucional de Derechos y Justicia ${ }^{10}$, lo cual para el tema de competencias y tipicidad en las acciones de control del órgano técnico implica un andarivel de sujeción obligatoria, pues, como lo rescata (Peña, 2010) lo dicho por el doctor Marco Morales, en clases de la Universidad Andina Simón Bolívar, respecto al Estado y la Constitución, indica, “...en el estado constitucional el poder está sometido a una sola constitución...", es decir, implica un sometimiento a la ley vigente y que desde el punto de vista formal y material, esto lleva a enlazarla con una de las características del ejercicio de las competencias como lo definieron tanto (Trujillo, 1994) y (Ávila, 2009), quienes coinciden, en que las potestades de los órganos se ejercen con límites, los cuales están establecidos en el derecho, y que en el caso de atentarlos, es obligación del estado subsanarlos aplicando la Constitución. En consecuencia, conforme lo concluye (González, 2013) y lo desarrolla (Ávila, 2009), el límite y fin del Estado son la protección de los derechos de las personas, por ello (Escobar, 2018) dice, que la legislación nacional debe abrirse a la aplicación directa e inmediata de la legislación internacional de protección de los derechos ${ }^{11}$, esto de parte de todas las funciones constituidas en el Estado.

Ahora en lo que se refiere a la tipificación de las infracciones sujetas a la potestad determinadora de responsabilidades de la Contraloría General del Estado, esta se debe fundamentar con el principio de legalidad ${ }^{12}$ y su alcance constitucional y su relación con la doctrina, del cual como lo definen García de Enterria, y Fernandez, (2006), de cuya correlación se puede determinar que, es una facultad que se otorga por ley, con límites previos, y que sin su configuración la administración no puede actuar; se advierte entonces, que para el proceso de control, el equipo auditor debe estar facultado expresamente para el efecto, como elemento primario, el cual inicia en la emisión del plan anual de control, que permite la emisión de la orden de trabajo, que está constituida por, objetivo general de la auditoría, alcance del trabajo, presupuesto

de recursos y tiempo; e, instrucciones específicas ${ }^{13}$; con lo cual se convierte en el límite de la acción de los auditores.

\footnotetext{
$9 \quad$ Art. 18.- Alcance y ejecución de la auditoría gubernamental.- El control externo que realizará la Contraloría General del Estado se ejercerá mediante la auditoría gubernamental y el examen especial,

10 Constitución de la República del Ecuador “Art. 1.- El Ecuador es un Estado constitucional de derechos y justicia,..."; recuperado de https:// www.oas.org/juridico/pdfs/mesicic4_ecu_const.pdf, 6 de junio 2021.

11 Constitución de la República del Ecuador (2008), art. 417, 426 y 427.

12 Constitución de la República del Ecuador “Art. 226.- Las instituciones del Estado, sus organismos, dependencias, las servidoras o servidores públicos y las personas que actúen en virtud de una potestad estatal ejercerán solamente las competencias y facultades que les sean atribuidas en la Constitución y la ley..."; recuperado de https://www.oas.org/juridico/pdfs/mesicic4_ecu_const.pdf , 6 de junio 2021.

13 Contraloría General del Estado Acuerdo 016-CG-2001.
} 
Por ende, por el referido principio de legalidad, la emisión de la orden de trabajo se constituye en un elemento de validez del proceso, es decir, que no va contra el derecho (Islas, 2009, Bhucely, 2015), requerimiento que ha sido desarrollado por la Corte Nacional del Ecuador (Recurso de Casación, 2016), que dictó:

“...el principio de legalidad o de legitimidad significa que la actuación de la Administración Pública está subordinada a la ley, por lo que debe respetar las formalidades previstas en ésta. En caso de verificarse que el acto administrativo no cumplió con éstas, los jueces, en ejercicio de su potestad jurisdiccional, pueden declarar su nulidad".

Como se puede advertir las actuaciones de los equipos de control deben estar planificadas y su ejecución amparada en el principio de legalidad, pues, por la supremacía del poder del Estado este debe estar limitado, ya que como lo dice (Escobar, 2016), impide que se desborde y prevengan en contra de la arbitrariedad.

Ahora, en lo que se refiere a la arbitrariedad, para contextualizar esta definición centra su pertinencia en la materia administrativa, conforme el Código Orgánico Administrativo ${ }^{14}$ (COA), que desarrolla varios principios, derechos y deberes a favor del administrado y por ende tutelas, entre los cuales se identifica el principio de juridicidad ${ }^{15}$, el cual señala que las actuaciones administrativas deberán someterse a la Constitución, a los instrumentos internacionales, a la ley, a los principios, a la jurisprudencia aplicable, paralelamente, es exigible que las acciones se ejecuten observando el principio de interdicción de la arbitrariedad establecido en el artículo 18 (COA), con lo que, los organismos que conforman el sector público deberán emitir sus actos conforme estos principios y no podrán hacer interpretaciones arbitrarias, esto enmarcado en los principios de seguridad jurídica y confianza legítima, conforme lo señala el artículo 22 del citado cuerpo normativo, en consecuencia las administraciones públicas actuarán bajo los criterios de certeza y previsibilidad y que los derechos de los administrados no podrán ser afectados por errores u omisiones de los servidores públicos en los procedimientos administrativos.

En ese contexto se entiende entonces, que la no arbitrariedad en la administración es un principio de tutela a los ciudadanos de recibir una buena administración pública (Rodríguez, 2014); en términos de derecho, colegiremos entonces, que es una obligación de los servidores públicos de no actuar de manera opuesta a la justicia, es decir, con injusticia, que se entiende como, la falta o la ausencia de la justicia en un comportamiento, un acontecimiento, hecho o accionar (Ucha, 2013).

14 Promulgado en el Registro Oficial Segundo Suplemento No. 31 de 7 de julio del 2017, vigente desde el 7 de agosto del 2018.

15 Código Orgánico Administrativo (art. 14) 
Por ende, en las acciones de control es pertinente aplicar cada principio, tutela y acción que en nuestra Constitución se desarrollan como garantía del debido proceso ${ }^{16}$; e insistiendo que como premisa principal y obligatoria de parte del equipo de control es el cumplimiento de estos, como lo manda los artículo 31 numeral 37 y 79 de la (LOCGE) ${ }^{17}$, además, que se debe aplicar los principios y deberes del derecho administrativo en lo que corresponden a las actuaciones de la administración pública y que se constituyen en garantías y derechos de los funcionarios y servidores públicos auditados, esto sustentado bajo la interpretación pro homine que implica la protección de derechos (Escobar, 2018).

Es por este razonamiento que se vuelve obligatorio su cumplimiento, pues, como lo sostiene (Benalcazar, 2007) en el control contencioso administrativo, se busca que la Administración se someta al Derecho, a la vigencia y eficacia de los derechos de los administrados, es decir, se debe tener siempre presente la presencia del cumplimiento de la tutela de la legalidad, la cual tiene importancia, pues, su incumplimiento y falta de aplicación en el proceso de control, tiene como efecto su no validez por inobservancia de estas garantías y tutelas recogidas dentro del debido proceso; por ende, las mencionadas acciones son obligatorias y no pueden ser omitidas por los auditores de Contraloría, al punto, que, de incurrirse en este incumplimiento, estas violaciones provocaran que el informe no sea aprobado (Consultas Jurídicas, 2011).

En consecuencia, este incumplimiento atenta contra el estado constitucional de derechos y justicia, lo cual también es recogido en nuestra jurisprudencia, la Corte Constitucional en el periodo de transición determinó en sentencia:

\begin{abstract}
"El primero de los subderechos del debido proceso es el deber de las autoridades administrativas o judiciales de garantizar el cumplimiento de las normas o lo derechos de las partes; constituye un principio fundamental para garantizar la defensa del Estado constitucional de derechos y justicia y una garantía indispensable para evitar la arbitrariedad en las decisiones y resoluciones de las autoridades administrativas o judiciales." (035-12-SEP, 2010).
\end{abstract}

Finalmente, el cumplimiento de los principios y deberes del derecho administrativo en lo que corresponden a las actuaciones de la administración pública se constituyen en garantías y derechos de los auditados, es decir, funcionarios y servidores públicos, pues su incumplimiento acarrea la nulidad del proceso.

\footnotetext{
16 Art. 76 de la Constitución de la República. Recuperado https://www.oas.org/juridico/pdfs/mesicic4_ecu_const.pdf

17 Art. 31.- Funciones y atribuciones. - La Contraloría General del Estado, además de las atribuciones y funciones establecidas en la Constitución Política de la República, tendrá las siguientes: (...) 37. Observar los derechos constitucionales individuales y las garantías del debido proceso en los informes que emita;..."
} 


\section{Legalidad de los informes aprobados durante la emergencia sanitaria}

Para el inicio de una acción de control es imperativo y elemento sine qua non la emisión de la orden de trabajo, pues al ser un documento donde se detallan por escrito las instrucciones para el proceso de control, y se designan al jefe de equipo, al auditor supervisor y a los auditores operativos ${ }^{18}$, y conforme el Manual de Auditoría Financiera Gubernamental, dispone que este instrumento contendrá los siguientes elementos: "Objetivo general de la auditoría. Alcance del trabajo. Presupuesto de recursos y tiempo. Instrucciones específicas"; por ende, la orden de trabajo se constituye en la presentación del Órgano de Control de su equipo de auditoría y notificación del inicio de las acciones de control, y además con la cual se requiere la acceso a la Entidad Auditada, y la disponibilidad de los Servidores Públicos; este documento se caracteriza por la precisión ${ }^{19}$, además marca la naturaleza jurídica del control, que legitima la determinación de la potestad legal de la acción de auditoría, que debe tener limitaciones en sus actividades conforme lo manda el artículo 14 del Reglamento a la Ley Orgánica de la Contraloría General del Estado (RLOCGE). En el control de contratación pública no se debe confundir la competencia otorgada versus su naturaleza legal, ya que auditoria en Contratación Pública analiza la actividad administrativa, financiera; $y$, operativa, violentando la tipicidad del artículo 14 LOSNCP, lo cual conduce a confusión en la individualización de las competencias, y por ende, el ejercicio del derecho a la defensa; es decir afecta a la objetividad y respeto a la legalidad, lo cual lleva a limitar la independencia del auditor, evidenciando graves consecuencias en los resultados de la auditoría (Lawrence, 1971), así como carencia del cumplimiento de sus objetivos, y que, finalmente, conlleva a que se produzcan acciones de control erradas o se actúe en supra posiciones de competencias (Flores, 2016).

A estas inobservancias previas debemos sumar otras violaciones legales en el procedimiento de aplicación de la acción de control, así tenemos notificaciones forzosas a los auditados, suspensión de plazos que afectan la caducidad de competencias, lo cual finalmente llegan a generar vicios de validez de las acciones de control. Por ende, se colige que es obligatorio para los servidores públicos en general aplicar los principios y deberes del derecho administrativo, ya que estos se constituyen en garantías y derechos de los ciudadanos, en el caso del control gubernamental, los auditados. Su incumplimiento acarrea nulidad del proceso, por ende, en una sanción de ineficacia que afecta a los actos procesales realizados con falta de alguno de los requisitos previstos por la ley para su validez (Colombo, 1998).

18 MANUAL DE AUDITORIA FINANCIERA GUBERNAMENTAL Capítulo II, punto 4

19 CODIGO: PAG. - 03GRUPO: Relacionadas con la Planificación de Auditoria Gubernamental 


\section{Notificación forzosa}

Como parte de las garantías del debido proceso en general, el cumplimiento en debida forma de las notificaciones de las acciones de control permite ejercer el derecho a la defensa ${ }^{20}$, así como también garantizar la tutela judicial efectiva, y seguridad jurídica (Escobar, 2016). La notificación debe ser presencial mediante oficio ${ }^{21}$, sin que para los procesos de control en la (LOCGE) y (RLOGCE) se determinen la posibilidad del uso de medios electrónicos; por lo tanto las notificaciones de los tres momentos procesales (inicio, resultados provisionales y convocatoria lectura de borrador) se constituyen en una garantía del debido proceso y por lo tanto en contrapeso al poder del Estado, por ello es el Jefe de Equipo el responsable del cumplimiento de esta garantía ${ }^{22}$, sin olvidar que es una función y atribución del Órgano de Control, artículo 31 numeral 37 (LOCGE) en concordancia con el 19 del Acuerdo 059-CG-2018, por ende, de incurrirse en este incumplimiento, esas violaciones provocaran que el informe incluso no sea aprobado (Consultas Jurídicas, 2011).

Durante las restricciones de movilidad por la emergencia sanitaria, el Órgano de Control ejecutó notificaciones por medios electrónicos, sustentando únicamente ese procedimiento en el Acuerdo 009-CG-2020, el cual se contrapone a las reglas de notificación del (COA) como se muestra en la Tabla 3.

Tabla 3. Restricciones.

\section{CÓDIGO ORGÁNICO ADMINISTRATIVO}

\section{ACUERDO 009-CG-200}

Al comparecer al proceso, determinar dirección de Fechay hora envió correo electrónico habilitada

Permitirá tener constancia de transmisión y recepción Correo o casilla electrónicos. de su contenido.

Designación de domicilio electrónico "voluntaria, Sin perjuicio de que haya o no revisado por el expresa, clara y precisa" destinatario.

Designación domicilio electrónico para cada proceso Utiliza un mismo domicilio electrónico

20 Art. 91 Ley Orgánica de la Contraloría General del Estado, recuperado https://www.contraloria.gob.ec/WFDescarga. aspx?id=2207\&tipo=tradoc

21 Art. 25 Reglamento Ley Orgánica de la Contraloría General del Estado, recuperado https://www.contraloria.gob.ec/WFDescarga. aspx?id=2207\&tipo=tradoc

22 Art. 18 Reglamento Sustitutivo para la Organización, Funcionamiento y Dependencia Técnica y Administrativa de las Unidades de Auditoría Interna de las Entidades que Controla la Contraloría General del Estado, recuperado https://www.contraloria.gob.ec/WFDescarga. aspx?id=2207\&tipo=tradoc 
Tampoco el instrumento define que se consideran medios electrónicos y telemáticos, tales como una página web y el correo electrónico (Salgado, 2002). Las notificaciones por ese medio no constituyen legalmente domicilio oficial de notificación, ya que la dirección de correo electrónico, ha de ser previamente signado como domicilio electrónico, esto, según el artículo 172 del (COA)리 y obligatoriamente signada de forma voluntaria, expresa clara y precisa (Thomson, 2018), y con el elemento fundamental, que cuando la notificación constituya un hecho de gravamen para el administrado, la primera actuación administrativa debe ser personal². Por lo tanto, la administración pública no puede arbitrariamente tomar una casilla electrónica de otro repertorio establecido para el ingreso de la declaración de patrimonio, en consecuencia, estas notificaciones se ejecutaron en un domicilio electrónico forzoso, aclarando, que en la legislación no existe autorización para que los órganos públicos puedan obtener una dirección de inscripción forzosa- la información que permita tener por constituido de oficio el domicilio procesal electrónico (Gaston, 2017).

Ahora es importante entender, que la transmisibilidad se configura al momento en que la entidad y/o administración remite el mensaje o dato electrónico al interesado o administrado, cuando la notificación sale del sistema de (CGE) con dirección al sistema de información o red electrónica del interesado. Después de lo cual, conforme el artículo 11 de la Ley de Comercio Electrónico, se entiende por recepción el momento que ingresa al domicilio electrónico previamente señalado para el efecto, y que "debe ser regularmente utilizado por el administrado" (Lilo, 2021), ratificando entonces, que la notificación debe trascender el hecho de una mera formalidad ${ }^{3}$ como lo ha dicho la Corte Constitucional del Ecuador ${ }^{4}$, ya que, por la relevancia que implica procedimentalmente la notificación esta debe perfeccionar para que sea eficaz.

Por lo tanto, cuando no se ha fijado en el proceso de juicio de responsabilidades un domicilio electrónico exclusivo para este proceso, la notificación no es eficaz y por ende no genera efectos jurídicos de notificación exitosa, pues esta "eficacia propone dentro de la notificación electrónica la posibilidad que los actos administrativos sean oponibles y generar efectos legales hacia sus administrados" (Amaris, 2009).

En cuanto a la receptibilidad, el Reglamento para las Notificaciones de Contraloría General del Estado, genera contradicción a una norma superior con rango de Orgánica (COA), pues (CGE) solo requiere tener la constancia del envió y no de la recepción, lo cual es ilegal e improcedente; pues la norma jerárquicamente superior requiere la recepción, que constituye tener el respaldo

$1 \quad$ Art. 172.- Comparecencia. La persona interesada, al momento de comparecer al proceso, determinará donde recibirá las notificaciones. Serán idóneos: 1. Una dirección de correo electrónico habilitada..." Código Orgánico Administrativo. Consultado 8 de octubre 2020 https://www. fielweb.com/Index.aspx?157Rabf6ik658\#app/buscador.

2 COA Art. 164.- “(...) La notificación de la primera actuación de las administraciones públicas se realizará personalmente (...)

Corte Constitucional del Ecuador, para el periodo de transición, sentencia 240-12-CC, caso 0165-09-EP

Corte Constitucional del Ecuador, sentencia 161-14-SEP-CC; caso 0542-13-EP 
de la "recepción del mensaje mediante un acuse de recibo para que éste surta efectos" (Weffer, 2018), en esa línea, la notificación para su perfeccionamiento debe contener tres elementos; momento y lugar de emisión, recepción, y acuse de recibo" (Weffer, 2018).

Así como se establece un obstáculo al administrado de denegación y acceso a la justicia y limitando la defensa, así la Corte Constitucional ya se ha pronunciado al respecto, en la Sentencia 117-14-SEP-CC; caso 1010-11-EP, ha determinado:

"La falta de notificación evitó que la accionante realice las actividades encaminadas a propiciar su defensa dentro del proceso, hecho que ha identificado la jurisprudencia constitucional como constitutivo de indefensión, lesivo de los derechos del debido proceso y a la defensa. En tales circunstancias, correspondía a la Sala desplegar la actividad necesaria para remover los obstáculos que evitaban que las formas procesales cumplan con su fin dentro del respectivo proceso o actuación. La Sala no garantizó el derecho a la defensa de la legitimada activa, pues en lugar de procurar remover los obstáculos administrativos, fácticos o de cualquier otra índole para garantizar su ejercicio, omitió solicitarle a la accionante que señale el casillero judicial para notificaciones posteriores, si consideraba que la dirección de correo electrónico provista por ella era insuficiente para tal efecto. En consecuencia, los jueces de la Sala Especializada de lo Civil, Mercantil, Laboral y Materias Residuales de la Corte Provincial de Justicia de Los Ríos vulneraron el derecho a la defensa de la accionante, consagrado en el artículo 76 numeral 7 literales a, b, c y h de la Constitución de la República." (Lo subrayado me corresponde).

En consecuencia, el Reglamento de Notificación está dejando en indefensión a los administrados, y vulnera el debido proceso desarrollado en la Constitución de la República, artículos 76. 7. h. Principio de contradicción, 76. 7. c. Derecho a ser escuchado en igualdad de condiciones, 76. 7. b. Derecho a contar con tiempo y medios adecuados para la defensa; y, 76. 7. a. Derecho a la defensa en todas las etapas procesales, así lo recoge la sentencia de la Sala de lo Mercantil de la Corte Provincial de Pichincha en el proceso 17981-2016-00675, la cual hace referencia a antecedentes jurisprudenciales de la Corte Constitucional5; lo cual también ha sido ratificado mediante sentencia No. 220-14-SEP-CC de la Corte Constitucional, publicada en el Registro Oficial No. 438-Tercer Suplemento de 13 de febrero de 2015 y otros ${ }^{6}$ que han sido recogidos y analizados por (Escobar, 2018); en consecuencia, esas notificaciones afectaron a la legalidad de la acción de control y por ende sus resultados que se constituyen en violatorios al derecho de los auditados al debido proceso.

5 Sentencias Corte Constitucional: 231-16-SEP-CC DE 2016/07/20; CASO 2234-13-EP; sentencia 228-16-SEP-CC de 2016/07/20; caso 1460-15EP; sentencia 227-16-SEP-CC de 2016/07/20; caso 1318-15-EP.

6 Corte Constitucional del Ecuador dentro de los casos Nros. 1334-15-EP, 1469-12 EP, 1705-13-EP, 0542-15-EP, 1816-11-EP, 1113-15-EP. 115614-EP, 0431-15-EP, 0359-12-EP; Y, 0435-12-EP 


\title{
Suspensión de los plazos
}

Con la promulgación del Código Orgánico Administrativo (COA), en la legislación ecuatoriana, se han incorporado y reconocido diferentes cambios los cuales, no solo son estructurales, sino, que fundamentalmente se han referido al fortalecimiento del principio del in dubio pro administrado, así como, a la ampliación y mejora de las garantías y derechos de las personas interesadas, o administrados en los procesos frente a la administración pública, en cierta forma, esto viene hacer, una reducción al poder y exorbitante posición del Estado sobre los ciudadanos. Estas tutelas además de estar estrechamente relacionadas con los derechos constitucionales, y la convencionalidad, obliga al Estado o Administración, a respetar el debido proceso administrativo, como obligación de las entidades y órganos de la administración pública, reconocida en el poder legislativo, poder ejecutivo, poder judicial, poder de control social; $y$, poder electoral (Cuchimarca, 2018); pero fundamentalmente en los órganos pertenecientes a la administración pública central, identificada en el artículo 45 del COA ${ }^{8}$.

Durante la emergencia sanitaria, el Órgano de Control', por intermedio del Contralor General del Estado, en su capacidad normativa, potestad que es regulada en el artículo 212.3 de la Constitución de la República, y que, se le reconoce inclusive desde la entidad rectora de la Planificación Nacional, que Contraloría, es el órgano que "produce innumerables regulaciones de aplicación para la administración, que suponen esfuerzo, tiempo y recursos para ser cumplidas" (Senplades, 2015), en ese contexto emitió el Acuerdo No. 007-CG-2020 de 17 de marzo de 2020, en el que su parte principal dispuso:

\begin{abstract}
“(...) Suspender todos los plazos y términos que se encuentren discurriendo desde el martes 17 de marzo de 2020, dentro de todos los procedimientos administrativos de este organismo técnico de control relacionados con la ejecución de la auditoría gubernamental y exámenes especiales, aprobación de informes de auditoría gubernamental, predeterminación y determinación de responsabilidades, recaudación y coactivas, así como en aquellos referidos a la entrega de documentación, respuestas a los pedidos de los equipos auditores, presentación de recursos o subsanación y calificación y registro de firmas auditoras privadas, los que se reanudarán una vez que las autoridades del Gobierno Nacional establezcan, forma oficial, la finalización de las medidas
\end{abstract}

\footnotetext{
7 Art. 225 de la Constitución de la República. Consultado 8 de octubre 2020 https://www.fielweb.com/Index.aspx?157Rabf6ik658\#app/ buscador

8 Art. 45.- Administración Pública Central. El Presidente de la República es responsable de la administración pública central que comprende:1. La Presidencia y Vicepresidencia de la República2. Los ministerios de Estado3. Las entidades adscritas o dependientes4. Las entidades del sector público cuyos órganos de dirección estén integrados, en la mitad o más, por delegados o representantes de organismos, autoridades, funcionarios o servidores de entidades que integran la administración pública central. Código Orgánico Administrativo. Consultado 8 de octubre 2020 https://www.fielweb.com/Index.aspx?157Rabf6ik658\#app/buscador.

9 "Art. 211.- La Contraloría General del Estado es un organismo técnico encargado del control de la utilización de los recursos estatales..." Constitución de la República. Consultado 8 de octubre 2020 https://www.fielweb.com/Index.aspx?157Rabf6ik658\#app/buscador.
} 


\section{restrictivas en el marco del Estado de Excepción por calamidad pública en todo el territorio nacional, lo cual será comunicado a través del portal web institucional www.contraloria.gob.ec(...)"10 (p 1); (Lo subrayado y negrita me pertenece).}

Como se puede advertir el Contralor General del Estado, en su capacidad de potestad reguladora emitió una directriz general, pero secundaria en los procesos normativos de las acciones de auditoría; no obstante, se puede colegir que dicha capacidad ha puesto en conflicto la validez de los términos de la ejecución de la acción de control, que se encuentran dispuesto en la LOCGE, generando un conflicto de jerarquía de la norma, esto bajo el criterio de la forma y grado $^{11}$, así la norma Parlamentaria tiene siempre carácter general y fundamentalmente fuerza de ley, mientras que los otros órganos no gozan de fuerza de Ley, de modo que en caso de existir conflictos entre ellas, la de mayor jerarquía priman sobre las de rango inferior (Castillo, 2012), de esta manera el Acuerdo de Contraloría General del Estado, violenta el principio de jerarquía que como indica (Otaduy, 2006) sigue siendo el único principio de estructuración del ordenamiento.

Este hecho como lo sostiene (Kelsen, 1969) violenta derechos constitucionales o derechos humanos-que la Constitución garantiza a las personas-y las reglas de estructuración y actuación, así esto se evidencia en el incumplimiento del debido proceso y ejercicio de competencias dentro de la acción de control, por ejemplo, generando incumplimiento al plazo del artículo 26 de la Ley Orgánica de la Contraloría General del Estado ${ }^{12}$, en lo que corresponde al tiempo para la aprobación del informe, ciento ochenta días contados desde la emisión de la orden de trabajo, mismo que es improrrogable; es decir, el Acuerdo no puede suspender la facultad de control, por ende durante ese periodo de tiempo la potestad de control feneció por fatal (Caducidad Competencia Contraloría General del Estado, 2017). Hay que considerar, que, "la obligatoriedad de los plazos configura un rigorismo procesal, atenuado sólo por dos caracteres propios del sistema: a) la posibilidad de ampliar los plazos (prorrogabilidad) y b) el hecho que los plazos no revistan, en principio, carácter perentorio o fatal" (Cassagne, 1994), además, que, en esa línea principalmente, se debe tener en cuenta, que, "un plazo fatal, no se puede interrumpir ni suspender, por cuanto busca la eficiencia en la actividad administrativa" (Aguado, 1999), lo cual también es recogido en nuestra jurisprudencia (Nulidad Resolución por Caducidad, 2017), que indica en el considerando cuarto "El plazo no se suspende ni se interrumpe, sino que desde que comienza a correr el tiempo se sabe cuándo caduca la acción”.

\footnotetext{
$10 \quad$ Acuerdo 007-CG-2020, Contraloría General del Estado.

11 Ruiz, M. (2015). Derechos Fundamentales Constitucionales y Derechos Humanos Internacionales. Compostela: Universidad de Santiago de Compostela.

12 Art. 26 (...) Estos informes serán tramitados desde la emisión de la orden de trabajo de la auditoría, hasta la aprobación del informe en el término máximo de ciento ochenta días improrrogables. Ley Orgánica de la Contraloría General del Estado. Consultado 8 de octubre 2020 https://www.fielweb.com/Index.aspx?157Rabf6ik658\#app/buscador.
} 
Se advierte en la práctica, que los equipos de control y auditoría de la Contraloría General del Estado, pese a la suspensión hicieron caso omiso, y en plena restricción y desarrollo de la emergencia sanitaria por COVID-19, por medios digitales - correos electrónicos, realizaron requerimientos de información, notificaciones de inicio, resultados provisionales y convocatorias a lectura de borrador, incumpliendo el Acuerdo 007-CG-2020 y por ende el debido proceso con lo cual se advierten excesos y riesgos de abuso o desbordamiento de la autoridad del Estado (Gómez, 1992), ante lo cual se determina, que la actividad de la Administración Pública rompe los criterios de objetividad, eficacia y eficiencia, y primordialmente el respeto de los derechos de los ciudadanos (García de Enterria, 2001).

De lo cual se colige, que existe excesos o riesgos de abusos, que, atentan a los derechos del debido proceso de los sujetos de las acciones de control auditados o administrativos; pues amenazan inminente al derecho a las garantías jurisdiccionales previstas en el artículo 76 ibídem, numerales 1,7 letras a), b); y, c) de la norma superior, que garantizan a todos los ciudadanos de la Republica el cumplimiento del debido proceso y el derecho a la defensa, fundamentalmente, el de tener acceso a una preparación de defensa técnica, a tiempo y con los elementos, recursos y medios adecuados; así como también a la seguridad jurídica, reconocido en el artículo 82 de la Constitución de la República.

Es decir, los equipos de control Contraloría General del Estado, procedieron con acciones forzosas, sin contar con una fundamentación técnica y normativa, en una clara desviación y abuso del poder, amenazando así el derecho de poder ser escuchados en el momento oportuno y en igualdad de condiciones. Lo realizado por los equipos de control suponen para el administrado sendas cargas y obligaciones procesales que debe cumplir. Finalmente, debemos indicar que si no se cumplen las obligaciones y cargas procesales mencionadas se producen consecuencias desfavorables para quien debe cumplirlas, por lo cual se produce una Nulidad.

\section{CONCLUSIONES}

El proceso de control gubernamental aplicado por Contraloría General del Estado tiene como elemento de sostenibilidad y procedibilidad los principios de legalidad, seguridad jurídica, interdicción de la arbitrariedad, juridicidad y tipicidad, lo cual enlaza una conexión y dependencia del Sistema de Control Gubernamental con el Código Orgánico Administrativo. Por ende, toda actuación administrativa generada por los servidores públicos del Órgano de Control, debe observar jerárquicamente los derechos, garantías y tutelas constitucionales así como los principios del derecho administrativo, conforme a la derivación de la balanza de equilibrios y dependencia de modo inmediato entre ellas, inclusive con los del bloque de 
constitucionalidad; en consecuencia, bajo ningún motivo o elemento emergente estos se vuelven opcionales o alternativos, así entonces las reformas de los procedimientos contractuales en régimen de emergencia no constituyen circunstancias de justificación para su incumplimiento, y mucho menos de motivo para que el Control sobrepase la Constitucionalidad y la legalidad del su accionar y provoque sin justificativo jurídico elementos de gravamen en contra de los administrados sujetos a las variaciones reglamentarias del órgano rector de la contratación pública.

\section{REFERENCIAS}

Aguado, V. (1999). Prescripción y caducidad en el ejercicio de potestades administrativas. Barcelona: Marcial Pons

Amaris, J. R. (2009). Diseño de un procedimiento administrativo electrónico en Colombia. Revista Republicana, (7)

Ávila, L.(2020). ¿Una nueva normalidad o una nueva humanidad? Discurso para la post pandemia. PENSAMIENTO JURÍDICO POPULAR, 1-3

Ávila, R. (2009). Del Estado legal de derecho al Estado constitucional de derechos y justicia. Anuario de Derecho Constitucional Latinoamericano, XV, 1

Ballén, R. (1998). Derecho Administrativo Disciplinario. Bogota: Temis

Benalcazar, J. C. (2007). Derecho Procesal Administrativo. Quito: Abya-Yala

Bhucely, L. (2015). Activismo burocrático: la construcción cotidiana del principio de legalidad. Bogota: Universidad de los Andes

Bonnemaison, M. (2000). Mecanismos constitucionales de control del poder público. Valencia: Universidad de Carabobo

Cassagne, J. (1994). Derecho Administrativo, T II. Abeledo-Perrot

Castillo, M. (2012). La Norma Jurídica en el Sistema Legislativo Peruano. Derecho y Cambio Social, 2-3

Colombo, J. (1998). Los actos procesales. Santiago de Chile: Editorial Jurídica de Chile

Cuchimarca, J. (2018). División de poderes en la actual Constitución del Ecuador. Universidad Andina, 50

Del Pozo, C. (2005). Control difuso y procedimiento administrativo. Lima: Palstra Editores

Dromi, J. R. (2015). Derecho administrativo
Dromi, E. (1979). More on the acquisition of locative prepositions: An analysis of Hebrew data. Journal of Child Language, 6(3), 547-562

Escobar, G. (2018). El Control Gubernamental, Determinación de Responsabilidades Administrativas, Civiles e Indicios Penales. Quito: Tallpa

Escobar, G. (2016). La determinación de las responsabilidades administrativas como consecuencia del control gubernamental Obtenido de http://repositorio.uasb.edu. ec/bitstream/10644/5629/1/T2281-MDAEscobarLa\%20determinacion.pdf

Flores, D. (2016). Un encuentro con la auditoría gubernamental. Revista Lidera, 34-38

García, M. Y. F. (2006). El concepto jurídico indeterminado de" servicio esencial" en la Constitución española. Revista de Administración pública, (170), 325-338

García de Enterria, E. (2001). Curso de Derecho Administrativo, Tomo II. Madrid: Civitas

Gaston, B. y. (2017). El nuevo régimen de notificaciones electrónicas en el proceso judicial bonaerense. Buenos Aires: ElDial DC22BF

Gómez, C. (1992). El debido proceso como Derecho Humano. Mexico: Facultada derecho UNAM

Gordillo, A. (2017). Tratado de Derecho Administrativo y obras selectas. Buenos Aires: Fundación de Derecho Administrativo

González, F. (2013). El alcance del Estado constitucional de derechos y justicia. Revista de Jurisprudencia, 6-7

Granda, V. (2017). El Derecho de Control y su relación con las modificaciones constitucionales y jurpidicas en los modelos políticos y económicos en Ecuador. Quito: Universidad Andina 
Guanchez, A. (2012). La participación ciudadana, modelo de una democracia responsable. Caracas: Editorial Jurídica Venezola

Harold Koontz y Heinz Weihrich. (2007). Administración una perspectiva global. México: Mc Graw Hill Interamericana

Islas, R. (2009). Sobre el principio de legalidad. Anuario de Derecho Constitucional Latinoamericano, XV, 97-108

Kelsen, H. (1969). Teoría Pura del Derecho. Buenos Aires: Editorial Universitaria de Buenos Aires.

Lara, C. (2012). Mecanismos de control en la contrtación estatal como elemento de la lucha anticorrupción. Revista en contexto, 37-65

Lawrence, R. (1971). Procedimientos de Auditoría. New York: Lives of Literature, Lives of Literature - Modernist Authors

Marquez, D. (2005). Función Jurídica de Control de la administración pública. México: Universidad Autónoma de México Instituto de Investigación Jurídicas

Marienhoff, M. (1998). Tratado de Derecho Administrativo. Buenos Aires: La Ley Edición

Nulidad Resolución por Caducidad, 177741-20170151 (Sala Especializada de lo Contencioso Administrativo 10 de Mayo de 2017)

Otaduy, J. (2006). El principio de la jerarquia normativa y la Instrucción Dignitas connubil. Navarra: Universidad de Navarra

Peña, P. (2010). Estado Social y Constitucional de Derechos y Justicia. Derecho Ecuador.com, 1

Polit, C. (2011). Consultas Jurídicas. Consultas Jurídicas, 28-33

Recurso de Casación, 356-2013 (Sala de lo Contencioso Administrativo - Corte Nacional del Ecuador 19 de julio de 2016)
Robanlino, J. (2008). Crisis de los Principios Generales del Derecho Administrativo. Derecho Administrativo, 4

Rodríguez, J. (2014). La buena administración como principio y como derecho fundamental en Europa. Misión Jurídica, 25

Ruiz, M. (2015). Derechos Fundamentales Constitucionales y Derechos Humanos Internacionales. Compostela: Universidad de Santiago de Compostela

Salgado, T. (2002). Validez de las notificaciones electrónicas en la administración pública. La administración pública - intenet, 1-9

Senplades. (2015). La capacidad regulatoria del estado. Quito: Telegrafo EP

Thomson, R. (25 de Octubre de 2018). Validez de notificaciones electrónicas. Obtenido de AR/ DOC/1896/2018: http://www.ordenjuridico. gob.mx/Congreso/pdf/166.pdf

Trujillo, J. C. (1994). Teoría del Estado en el Ecuador. Quito: Corporación Editora Nacional

Ucha, F. (MArzo de 2013). Definiciones jurídicas ABC. Obtenido de URL: https://www. definicionabc.com/derecho/arbitrariedad.php

Valadés, D. (2006). El control del poder. México: Porrúa

Vásquez Ochoa, P. A. (2021). La eficacia de los medios de impugnación en la contratación pública ecuatoriana

Weffer, N. M. (2017). El uso de las notificaciones por correo electrònico en el sistema judicial y su rol en la administración de justicia venezolana. Iustitia Socialis: Revista Arbitrada de Ciencias Jurídicas y Criminalísticas, 2(3), 38-53 